\title{
H I Imaging Surveys: Gas and Galaxy Evolution in Different Environments
}

\author{
Jacqueline van Gorkom ${ }^{1}$ \\ ${ }^{1}$ Department of Astronomy, Columbia University, \\ New York, NY 10027, USA \\ email: jvangork@astro.columbia.edu
}

\begin{abstract}
Our understanding of the formation and evolution of galaxies and the large scale structure has advanced enormously over the last decade, thanks to an impressive synergy between theoretical and observational efforts. While the growth of the dark matter component seems well understood, the physics of the gas, during its accretion, removal and/or depletion is less well understood. Increasingly large scale optical surveys are tracing out the cosmic web of filaments and voids and mathematical tools have been developed to describe these structures and identify galaxies in specific environments. $\mathrm{H} \mathrm{I}$ imaging surveys begin to answer the question: how do galaxies get and lose their gas. The best evidence for ongoing gas accretion is found in the lowest density environments, while removal of gas in the highest density environments stops star formation and reddens the galaxies. Although current H I emission surveys are limited to redshifts less than 0.2 , this is where the LSS is best defined and much can be learned in the local universe.
\end{abstract}

Keywords. galaxies, evolution, ISM, large-scale structure

\section{Introduction}

Spectroscopic surveys of the nearby universe such as SDSS and 6dF show that galaxies trace out an intricate cosmic web of filaments, sheets, bubbles or voids and dense clusters at the intersections of filaments. Theoretically structure can be reproduced well by simulations of the growth of structure in dark matter, for example the millennium simulation. Galaxies grow by infall of gas in the halos and the merging of dark halos. Currently a major uncertainty in this process is the detailed physics of the gas. While the seminal paper on galaxy formation and evolution by White \& Rees 1978 assumed that gas would always be shock heated to the virial temperature of the halo, more recent simulations show that some of the gas has such a short cooling time that it never heats up to the virial temperature and gas may feed into galaxies along filaments of cold gas, so called cold mode accretion (e.g. Keres et al. 2005). Thus it has become very interesting to study the infall and removal of gas in detail. This can be done with $\mathrm{H} \mathrm{I}$ imaging instruments. I will show here some results of $\mathrm{H}$ I imaging surveys in different environments. Although this kind of work has so far mostly been done in the very low redshift universe, the future of this work is very bright indeed, as many $\mathrm{H}$ I imaging instruments will become operational in the coming years, instruments such as ASKAP, MeerKat, Apertif, ATA and EVLA, that are extremely well suited to do large surveys

\section{HI imaging of void galaxies}

The simulations and data show that structure evolves from a relatively smooth distribution at high redshifts to a cosmic web of filaments and sheets, surrounding mostly empty regions at low redshifts. Locally $95 \%$ of the volume is taken up by voids. Although 


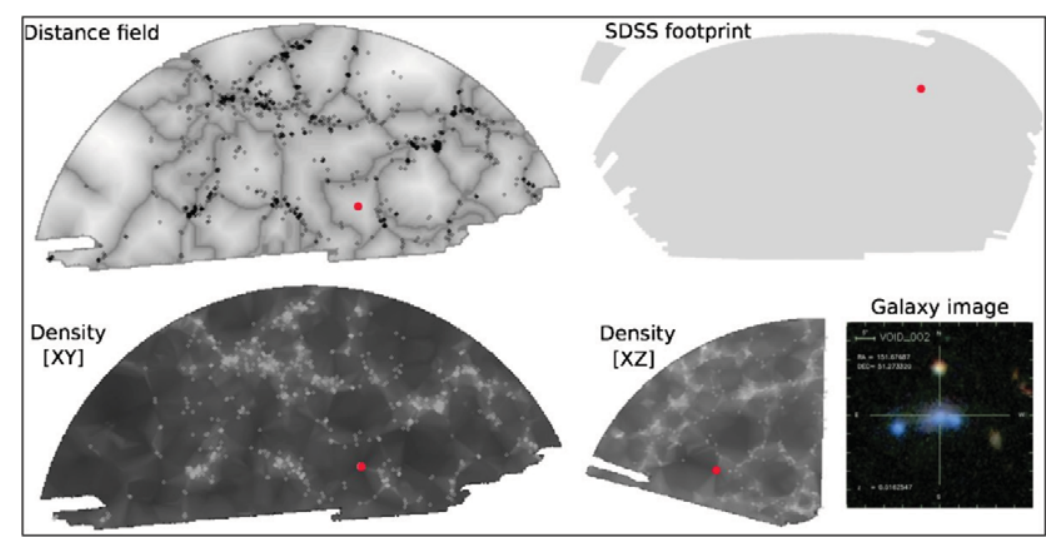

Figure 1. An example of a galaxy selected by geometry from SDSS. Top right: the SDSS footprint with the void galaxy indicated as red dot. Bottom left and center: DTFE density gray-scale maps in two mutually perpendicular slices intersecting at the galaxy location. Bottom right: galaxy image from SDSS. Top left contours of the DTFE density field in dark gray on top of the distance field.

voids appear to be mostly empty there are some galaxies in voids and these provide a unique opportunity to study galaxies in an earlier stage of their evolution, mostly undisturbed by the larger environment. Kreckel et al. 2011a have carried out an H I imaging survey of galaxies in voids selected from the SDSS. They use a new geometric method to find the deepest underdensities of the voids, a method which makes no a priori assumption about the shapes of the voids, and a method which recovers the structure on small scales as traced out by the galaxies. The methods involved, the Delaunay Tesselation Field Estimator (DTFE) to reconstruct a continuous density field from a discretely sampled galaxy distribution and the watershed void finder to find the deepest underdensities in the voids, are described in van de Weygaert \& Schaap 2009 and Platen 2010 respectively.

The survey selected galaxies in the deepest underdensity of 60 voids found in the SDSS. Those galaxies were observed with a new backend of the Westerbork Synthesis Radio Telescope (WSRT), which instantaneously probe a velocity range of $10000 \mathrm{~km} / \mathrm{s}$, thus not only each void galaxy got observed but also fore and background galaxies located in higher density environments, which form a natural control sample. Figure 1 shows an example of the location of selected void galaxies and control galaxies within the large scale structure. The main results of a pilot for this survey are presented in Kreckel et al. 2011a Void galaxies turn out to be small, mostly blue and relatively gas rich. They follow the usual scaling relations, such as the Tully Fisher relation and they have typical H I masses for their optical luminosity. It is the distribution of the gas that is most interesting. The gas disks are unusually large and several galaxies show evidence for ongoing cold gas accretion. Lastly, the star formation rate per HI mass appears to be slightly elevated in the lowest density regions, possibly stimulated by ongoing infall. One of the best cases of recent infall is a polar disk galaxy (Stanonik et al. 2009) shown in Figure 2 at left. The H I mass in the polar disk $\left(3 \times 10^{9} M_{\odot}\right)$ exceeds the stellar mass in the galaxy by a factor 3. No optical or UV counterpart is found in the H I polar disk. These observations seem to rule out a merger or tidal accretion of another galaxy, as a tidal interaction with this mass ratio would have destroyed the rotation in the stellar disk. The observed 

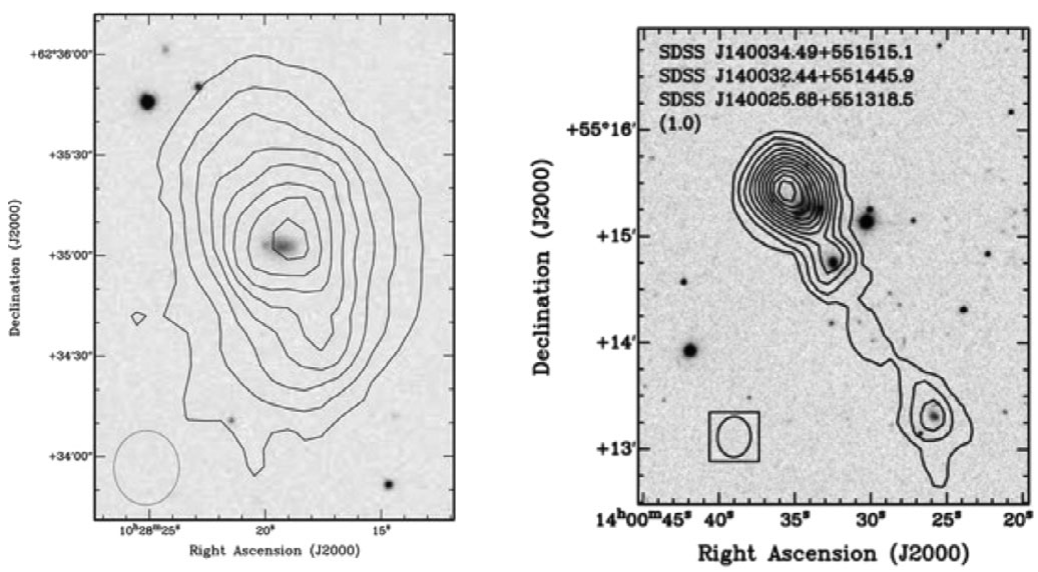

Figure 2. At left HI polar disk (contours) on SDSS (greyscale), at right HI filament on SDSS.

stellar disk is thin and rotationally supported. Thus it is more likely that the polar disk has been formed by ongoing cold gas accretion onto the galaxy. Several other galaxies in the survey show evidence of unusual kinematics in the H I. Another interesting result is shown in Figure 2 at right, a target galaxy seems to be located in a thin $\mathrm{H}$ I filament, in which two other galaxies are found. The preliminary results suggest that voids are good places to look for ongoing gas accretion.

This is confirmed in the local Tully void, where Kreckel et al. 2011b imaged the only known galaxy, KK 246, in HI. KK 246 has one of the largest H I disks known, with $\mathrm{D}_{H I} / \mathrm{D}_{\text {opt }}=5, \mathrm{M}_{H I} / \mathrm{L}_{B}=2.3$ and $\mathrm{M}_{\text {dynamical }} / \mathrm{L}_{B}=89$, hence it is also one of the darkest galaxies known. The H I disk is misaligned with the optical disk and some gas is found in the outer disk at forbidden velocities, which is another hint of recent infall.

There are other pieces of evidence that voids or possibly the outskirts of voids are inducive to gas infall. In a recent paper on some challenges to the standard LCDM scenario, Peebles \& Nusser 2010 point out that the larger disk galaxies in our local neighborhood, including M 101 and NGC 6946, are not found in the supergalactic plane, as expected from simulations, but instead in the much lower density regions, close to the local void. These same galaxies (M 101, NGC 6946) are mentioned in a paper by Kormendy et al. 2010 as a problem for hierarchical galaxy formation, as they are very massive, yet have no bulge at all. The question is how can these galaxies become so big without any evidence for past merging? Interestingly these are the very galaxies that show some of the best evidence yet for infall of H I (van der Hulst \& Sancisi 1988, Boomsma et al. 2008). Growth by gentle accretion of cold gas would explain the absence of a bulge despite the large size. Their location suggests yet again that it is in the lowest density regions where we may be seeing evidence for cold mode accretion.

\section{The other extreme, the center of Virgo}

Recently a new VLA H I imaging survey of selected galaxies in the Virgo cluster (VIVA, VLA Imaging of Virgo spirals in Atomic gas) has been completed Chung et al. 2009. This survey confirmed some already well known facts, e.g. that disk galaxies in the center of Virgo are ram pressure stripped to well within the optical disk. However 
many new things were learned as well. In addition to obtaining highly improved H I data, integral field spectroscopy has been done centered on the stripped part of the disks of selected galaxies to determine the star formation history and specifically the time at which star formation was quenched (Crowl \& Kenney 2008). Lastly detailed modelling has been done for selected galaxies (Vollmer 2009). This combined data set can now be used to trace the the orbital history of the galaxies, determine the onset of ram pressure stripping and assess the impact of the removal of cold gas on the star formation history and global color evolution of the galaxies.

\subsection{The onset of ram pressure stripping in the outer parts of the cluster}

Several lines of evidence are found that ram pressure stripping already occurs at large projected distances from the cluster center. For example a number of long one sided H I tails are found at projected distances of 0.6 - 1 Mpc to M87 (Chung et al. 2007). The tails are extended well beyond the optical disk on one side and point roughly away from M87, suggesting that they have been created by a global cluster mechanism. A rough estimate of the local ram pressure strength suggests that simple ram pressure stripping could indeed have formed the tails in all but two cases. At least three systems show H I truncation to within the stellar disk on the side opposite of the tail, providing evidence for a gas-gas interaction. The authors propose as most likely interpretation that these galaxies are on highly radial orbits, falling into the cluster for the first time. It shows that ram pressure stripping already starts at the virial radius of the cluster.

\subsection{Evidence for a dynamic and clumpy intra cluster medium}

A particularly interesting galaxy is NGC 4522 (Kenney et al. 2004), which is at a projected distance of $800 \mathrm{kpc}$ from M87. The galaxy has an H I disk that is stripped to deep within the optical disk. All observational evidence points to strong ongoing ram pressure stripping, yet its location in the far outskirts of the cluster would suggest that locally the ram pressure would be too low by a factor 10 for that. A possible explanation is that the M49 subcluster is falling into Virgo close to NGC 4522 and its ICM has stirred up the ICM of Virgo. This is consistent with a cosmological simulation of cluster formation by Tonnesen et al. 2007 who find a wide scatter in ram pressure strength at a fixed distance from the cluster center due to variation in the ICM density and the relative velocity between the galaxy and the ICM (Tonnesen \& Bryan 2008). There is other evidence for strong ram pressure stripping in the outskirts. Using integral field spectroscopy Crowl \& Kenney 2008 find that for several strongly stripped galaxies in the outskirts the time since last star formation is relatively short, less than the time it would take to travel from the center of Virgo to their current location. Those galaxies must therefore also have been stripped locally.

\subsection{The impact of ram pressure stripping on global color evolution}

The VIVA survey has provided us with a samply of galaxies in various stages of ram pressure stripping, for which the orbital history is well understood. This makes it possible to quantify the effect of ram pressure stripping on the evolution of the global colors of the galaxies. Figure 4 shows the location of the VIVA disk galaxies on a color magnitude diagram of the SDSS. The VIVA galaxies appear to be evenly distributed over the blue cloud, green valley and red sequence. The H I imaging combined with integral field spectroscopy teaches us a few new things. First we can compare the ratio of the sizes of H I disks to optical disks with the global colors of the galaxies. All the galaxies in the blue cloud have $\mathrm{H}$ I disks that are larger than the optical disks, the galaxies in the green valley have a range of values, and many look somewhat distorted, while all but two of 


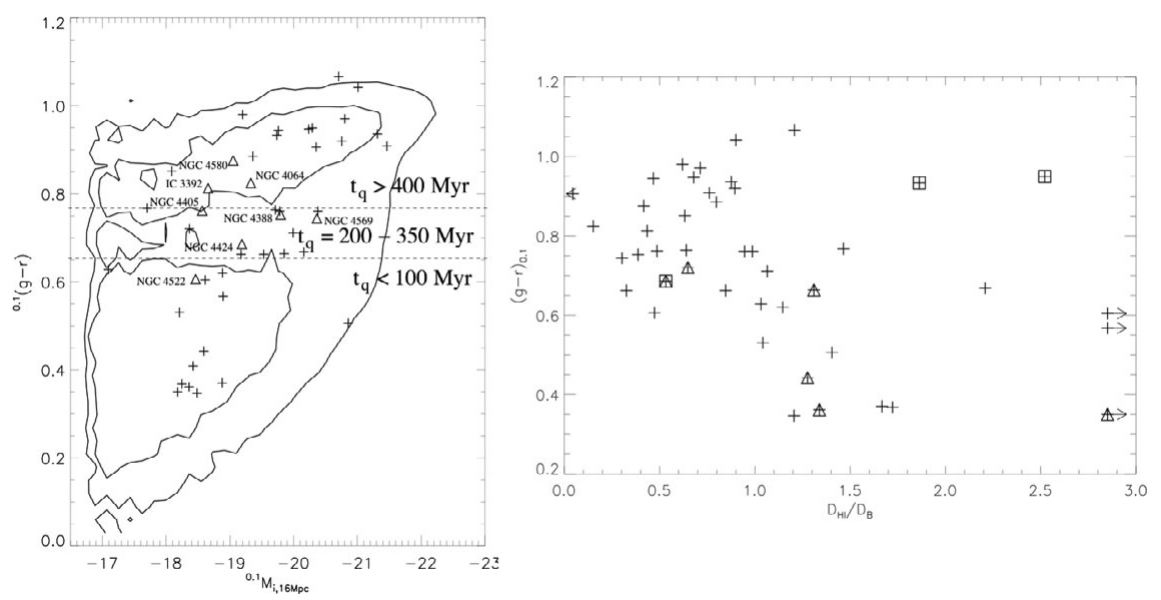

Figure 3. At left: VIVA galaxies (+) on top of SDSS (contours)in a color magnitude diagram. The dotted lines indicate the approximate position of the green valley. The quenching time for ram pressure stripped galaxies is indicated. At right: The HI diameter/ optical B diameter versus (g-r) color for the VIVA galaxies. Triangles are the long one sided HI tails found by Chung et al. 2007, squares are merging and interacting galaxies, from Crowl et al. 2011.

the galaxies on the red sequence have symmetrical H I disks that are much smaller than the optical disk (Figure 3). This suggests that somehow the presence of an extended H I reservoir plays a role in controlling the star formation rate in galaxies. The galaxies that get ram pressure stripped lose the H I in the outskirts, and as the stripping continues they enter the green valley. Once most of the gas has been stripped to well within the optical disk and at most a small, symmetric, H I disk is left the galaxies turn globally red. From a comparison of the quenching time we can derive that it takes a few $100 \mathrm{Myr}$ for a stripped galaxy to cross the green valley (Crowl et al. 2011).

\section{The future}

In the previous sections we have seen how $\mathrm{H}$ I imaging surveys may help us study how galaxies get and lose their gas. Most of the data obtained so far are for redshifts less than 0.1. A recent survey with the WSRT by Verheijen et al. 2010 of two galaxy clusters at $\mathrm{z}=0.2$ provides a first hint what the future will bring. The large field of view of the WSRT and the instantaneous velocity range of $18000 \mathrm{~km} / \mathrm{s}$ allows for the imaging of all galaxies in a huge volume of $5 \mathrm{Mpc} \times 5 \mathrm{Mpc} \times 18000 \mathrm{~km} / \mathrm{s}$. Hence not only the clusters but also the large scale structure in which they are embedded is imaged in $\mathrm{H} \mathrm{I}$ (Verheijen et al. 2010). The EVLA is being commissioned and it will soon be possible to probe instantaneously a range from $\mathrm{z}=0$ to $\mathrm{z}=0.18$ and eventually after the upgrade is complete, from $\mathrm{z}=0$ to $\mathrm{z}=0.45$. Until the SKA comes online the EVLA and MeerKat will be the best instruments to probe deeply in $\mathrm{H}$ I out to larger redshifts, while ASKAP and Apertif will be ideally suited to do large area imaging surveys to finally make an $\mathrm{H} \mathrm{I}$ image of the (local) Universe.

\section{Acknowledgments}

I am very grateful to Aeree Chung, Hugh Crowl, Kathryn Kreckel and Erwin Platen for pretty images and all my collaborators on clusters and voids for many stimulating 
discussions. This work was partially supported by NSF grant 1009476 to Columbia University.

\section{References}

Boomsma, R., Oosterloo, T. A., Fraternali, F., van der Hulst, J. M., \& Sancisi, R. 2008, A\& A, 490,555

Chung, A., van Gorkom, J. H., Kenney, J. D. P., \& Vollmer, B. 2007, ApJ 695, L115

Chung, A., van Gorkom, J. H., Kenney, J. P. D., Crowl, H., \& Vollmer, B. 2009, AJ, 138, 1741

Crowl, H. H. \& Kenney, J. P. D.2008 AJ, 136, 1623

Crowl, H. H., Chung, A., Kenney, J. P. D., van Gorkom, J. H., Schiminovich, D., \& Blanton, M. 2011, submitted

Kenney, J. D. P., van Gorkom, J. H., \& Vollmer, B 2004, AJ, 127, 3361

Keres, D., Katz, N., Weinberg, D. H., \& Dave, R. 2005, MNRAS, 363, 2

Kormendy, J., Drory, N., bender, R., \& Cornell, M.E., 2010 ApJ, 723, 54

Kreckel, K., Platen, E., Aragon-calvo, M. A., van Gorkom, J. H., van de Weygaert, R., van der Hulst, J. M., Kovac, K., Yip, C.-W., \& Peebles, P. J. E. 2011a, AJ, 141, 4

Kreckel, K., Peebles, P. J. E., van Gorkom, J. H., van de Weygaert, R., \& van der Hulst, J. M. $2011 \mathrm{~b}, A J$, in press

Peebles, P. J. E. \& Nusser, A. 2010, Nature, 465, 565

Platen, E. 2010, PhD thesis, Univ of Groningen

Stanonik, K., Platen, E., Aragon-calvo, M. A., van Gorkom, J. H., van de Weygaert, R., van der Hulst, J. M., \& peebles, P. J. E. 2009, ApJ, 696, L6

Tonnesen, S., Bryan. G. L. \& van Gorkom, J. H. 2007, ApJ, 671, 1434

Tonnesen, S. \& Bryan, G. L. 2008, ApJ, 684, L9

Verheijen et al. 2010, astroph:1009.0279

van de Weygaert, R. \& Schaap, W. 2009, in Lecture Notes in Physics, Vol 665, eds. Martinez, Saar, Gonzales, Pons-Borderia (Berlin:Springer), 291

van der Hulst, J.M. \& Sancisi, R., 1988 AJ, 95, 1354

Vollmer, B.2009, $A \& A$, 502, 427

White, S. D. M. \& Rees, M. J. 1978, MNRAS, 183, 341 